Article

\title{
Optimizing the Use of Secchi Depth as a Proxy for Euphotic Depth in Coastal Waters: An Empirical Study from the Baltic Sea
}

\author{
Hanna Luhtala * and Harri Tolvanen \\ Department of Geography and Geology, University of Turku, Turku FI-20014, Finland; \\ E-Mail: harri.tolvanen@utu.fi \\ * Author to whom correspondence should be addressed; E-Mail: hanna.luhtala@utu.fi; \\ Tel.: +358-2-333-6083; Fax: +358-2-333-5896.
}

Received: 30 September 2013; in revised form: 5 November 2013 / Accepted: 26 November 2013 / Published: 9 December 2013

\begin{abstract}
Potential zone for photosynthesis in natural waters is restricted to a relatively thin illuminated surface water layer. The thickness of this layer is often indirectly estimated by measuring the depth in which $1 \%$ of the photosynthetically active radiation entering the water remains. This depth is referred to as the euphotic depth. A coarser way to evaluate the underwater light penetration is to measure the Secchi depth, which is a visual measure of water transparency. The numerical relationship between these two optical parameters, i.e., conversion coefficient $m$, varies according to the changes in the optical properties of water, especially in transitional coastal waters. The aim of our study is to assess which is the most suitable criterion to base these coefficients on. We tested nine methods, seven of which were locally calibrated with our own in situ data from the optically heterogeneous Baltic Sea archipelago coast of SW Finland. We managed to significantly improve the accuracy of modeling euphotic depths from Secchi depths by using scalable and locally calibrated methods instead of a single fixed coefficient. The best results were achieved by using methods, either continuous functions or series of constants, which are based on water transparency values.
\end{abstract}

Keywords: Secchi depth; euphotic depth; method optimization; modeling accuracy; coastal waters; Baltic Sea 


\section{Introduction}

Underwater light is an important environmental variable because its low availability limits the photosynthetic activity, and thereby the primary production of the aquatic ecosystem [1]. Light is attenuated by water molecules, suspended particulate matter (SPM), colored dissolved organic matter (CDOM, yellow substances), and chlorophyll along with other photosynthetic pigments of living phytoplankton. Each of these components attenuates light from different wavelengths, and some components are primarily absorbers and some scatterers. For example, dissolved substances primarily absorb light, suspended particles scatter, and photosynthesizing cells do both [1].

Due to the efficient attenuation in natural waters, the illuminated surface layer, and consequently the potential zone for photosynthesis, is relatively thin. The thickness of the photosynthetically active water layer can be assessed by comparing the amounts of photoautotrophic production and heterotrophic consumption, but it can also be roughly estimated by measuring the amount of light in the water column, e.g., [1]. A common variable is the euphotic depth $\left(Z_{e u}\right)$, which is relatively easy and accurate to determine [2] by measuring the depth in which $1 \%$ of the photosynthetically active radiation (PAR, 400-700 $\mathrm{nm}$ ) entering the water remains.

Another way to evaluate the underwater light penetration is to measure the Secchi depth $\left(Z_{S D}\right)$, which is the depth in which a white Secchi disc disappears from sight. $Z_{S D}$ is a visual measure to provide a numerical variable that describes the clarity of the water [3]. The use of Secchi disc measurements is a traditional and common practice in marine observation and monitoring programs. It is an easy, inexpensive, and straightforward method, and thus often included in a field program-if not as a main focus of the particular study, at least as a background variable describing the prevailing conditions. The method is, however, rather coarse. Compared to direct PAR measurements, Secchi readings are more prone to measurement inaccuracies caused by, for example, surface roughness, general illumination conditions or human interpretation. The potential sources of error need to be acknowledged before making any comparisons.

The Secchi disc readings and underwater light sensors react differently to changes in the absorption/scattering balance, i.e., changes in the relative amounts of, for example, SPM and CDOM in water ([4] and references therein). Consequently, the relationship between the optical parameters, or in this case the $Z_{e u}: Z_{S D}$ ratio, varies according to the changes in the optical properties of water, e.g., [1,4-6]. The correlation between the parameters is, in its simplest form, expressed as follows:

$$
Z_{e u}=m \times Z_{S D}
$$

The reported values of the conversion coefficient, $m$, usually range from less than 1 up to 5 , or even 10 ([4] and references therein). Holmes [7], for example, suggests a factor of 3 to be a suitable general coefficient in turbid waters, but, at the same time, he adds that a higher factor would be better for more turbid waters, and a lower one for clearer waters.

Preisendorfer [3] has criticized the attempts to create coefficients to convert $Z_{S D}$ to $Z_{e u}$. He rationalizes that, if instruments for higher quality optical measurements are needed in assessing the factors, they should be used to measure the underwater light field directly. This is a valid statement. However, since underwater PAR measurements require specific instruments and knowledge, and 
despite their increasing popularity in routine ocean surveys, they are still performed less frequently than $Z_{S D}$ measurements. Instead, Secchi depth measurements continue to be a normal procedure within any marine sampling campaign. In addition, the history of the $Z_{S D}$ method is much longer than that of the modern optical measurement techniques; the first measurements were made already at the early 19th century [8]. Whenever and wherever Secchi disc measurements are the only optical data available, they are highly valuable, since they provide - as opposed to nothing - at least some information about the underwater light conditions.

All in all, $Z_{S D}$ data are much more plentiful on both spatial and temporal dimension than data on euphotic depths or attenuation coefficients [8]. By defining the quantitative linkage between $Z_{S D}$ and other parameters, the excessive, already existing, and continuously accumulating $Z_{S D}$ data can be utilized more efficiently. Holmes [7] pointed out that biologists may not need the same high degree of accuracy and precision required by optical oceanographers, and thus the $Z_{S D}$ conversions-despite their limitations - might be useful in certain study types. Moreover, in highly variable, constantly fluctuating coastal conditions, the absolute accuracy of point measurements can even be seen as rather irrelevant if they only represent very local and highly momentary conditions [9].

Due to the reasons mentioned above, researchers will undoubtedly continue to convert $Z_{S D}$ measurements to $Z_{e u}$ estimates. In some cases, such as the historic Secchi data dating before the availability of remote sensing data or underwater light sensors, the $Z_{S D}$ to $Z_{e u}$ conversion is the only way to obtain information about the euphotic depth. While it is advisable to use optical equipment whenever possible, it is still inevitable that $Z_{S D}$ will be, by far, the most common measure of underwater light conditions also in the future. Rather than trying to suppress the common practice, we find it important to aim at its optimization. The indirect conversion results provide more reliable information about the thickness of the productive layer than using the Secchi depth as such.

Geographical information systems (GIS) are widely utilized in marine research, especially in coastal areas. Spatial data and modeling methods add to the understanding of the marine ecosystems from the littoral to the benthic environments. Characteristic to the shallow coastal seas, the euphotic zone regulates the occurrence and depth distribution of seafloor habitats. For instance, in our study area, the euphotic seafloor area fluctuates approximately $100 \%$ from yearly minimum to maximum [10]. As $Z_{e u}$ is a key variable in any model concerning the marine environment, its use also in GIS modeling is increasing. This calls for optimized parameterization of the phenomenon, including improved understanding of the spatial characteristics of the water transparency and its driving forces.

In this paper, we compare alternative methods for determining the coefficients between $Z_{S D}$ and $Z_{e u}$, using our own in situ data from the Baltic Sea archipelago coast of SW Finland. The aim is to assess, which is the most suitable criterion to base the coefficient on. As it is vital to understand the importance of local empirical calibration, we do not present any general coefficients. Instead, we study our empirical data in detail and urge researchers to critically consider the most suitable conversion method for their particular study areas. The conversion procedure may be rather straightforward in oceanic offshore conditions, but we would like to pay more attention to transitional coastal environments with more varying water quality. 


\section{Material and Methods}

\subsection{In situ Measurements}

Both Secchi depth and euphotic depth data were collected by in situ sampling using a small (length $\sim 5 \mathrm{~m}$ ) boat. Water transparency was measured using a white Secchi disc, and the results were recorded by the same person with $0.1 \mathrm{~m}$ accuracy. In quantifying the euphotic depths, we used an instrument set of two LI-COR quantum sensors (LI-COR Biosciences, Lincoln, NE, USA), which register the amount of radiation $\left(\mu \mathrm{mol} \cdot \mathrm{s}^{-1} \cdot \mathrm{m}^{-2}\right)$ in the $400-700 \mathrm{~nm}$ wavelength area, i.e., spectrally integrated values of PAR. The amount of underwater light was measured with a spherical quantum sensor (model LI-193). Compared to measurements of downwelling PAR, scalar irradiance measurements are more practical in studies related to photosynthesis [1] and are not as sensitive towards changes in the solar elevation angle [11]. Simultaneously, terrestrial quantum sensor (model LI-190, cosine collector) was used to observe the changes in the incoming radiant flux above the sea surface and to mathematically normalize the flux in a way that the underwater measurements are comparable with each other. The measurements were performed between 08:00 and 19:00 h, local daylight saving time. The timings were considered appropriate concerning the summer season solar elevation angles at $60^{\circ} \mathrm{N}$ latitude.

The underwater measurements were started by recording PAR readings just below the sea surface, proceeding downwards with an interval of one meter. The maximum measurement depth was determined by the depth of the respective sampling station, however, never exceeding $20 \mathrm{~m}$. At the shallowest sampling station the measurement range was 0-5 m. At least three separate measurements were logged from every depth with LI-1400 data logger (LI-COR Biosciences, Lincoln, NE, USA). Outliers, which deviated more than $20 \%$ from the median of the particular depth, were removed and averages of the remaining measurements were used as the final values for every measurement depth. These light profiles were then used to calculate the lower limit of the euphotic zone according to the rule of $1 \%$ PAR penetration (for a detailed method description, please see also [12]).

In order to include variable underwater light conditions into the data, we conducted a field campaign that covers both the spatial and temporal changes in the optical properties of the coastal archipelago waters of the Baltic Sea. The campaign was conducted in the NE part of the SW-Finnish archipelago, which provides highly variable water quality conditions within relatively small distances. Optically, the Baltic Sea represents Case-2 waters with relatively high CDOM concentrations [13,14]. The SPM and chlorophyll values undergo great spatio-temporal variations within the study area $[12,15]$.

The campaign of the original training data included 11 sampling stations that were located within an area $45 \mathrm{~km}$ by $40 \mathrm{~km}$, with distances of 7-16 km separating adjacent stations (Figure 1). The stations were visited (with one exception) every three weeks from late April to early October in 2010, resulting in eight visits at each. In total, that adds up to 88 individual light profiles coupled with $Z_{S D}$ values.

To assess the applicability of the calibrated conversion methods, we also used independent testing data from year 2011. It included three networks of stations, located in the same sea area as the training data (Figure 1). Each network represented different archipelago zones, and comprised of 15-16 stations with smaller spatial intervals. The networks were built by stratified random sampling around some of 
the original stations from the previous year. First, we created a grid of 16 squares $(2 \mathrm{~km}$ by $2 \mathrm{~km})$, masked out all land areas and waters shallower than $10 \mathrm{~m}$, and then by random sampling created a station in each grid. Whenever there was an original station within the square, it was chosen and no new station was created. All the stations were visited twice: in early June (week 23) and early August (week 31). The total number of $Z_{S D}-Z_{\text {eu }}$ pairs was 94 .

Figure 1. The study area and the sampling stations.

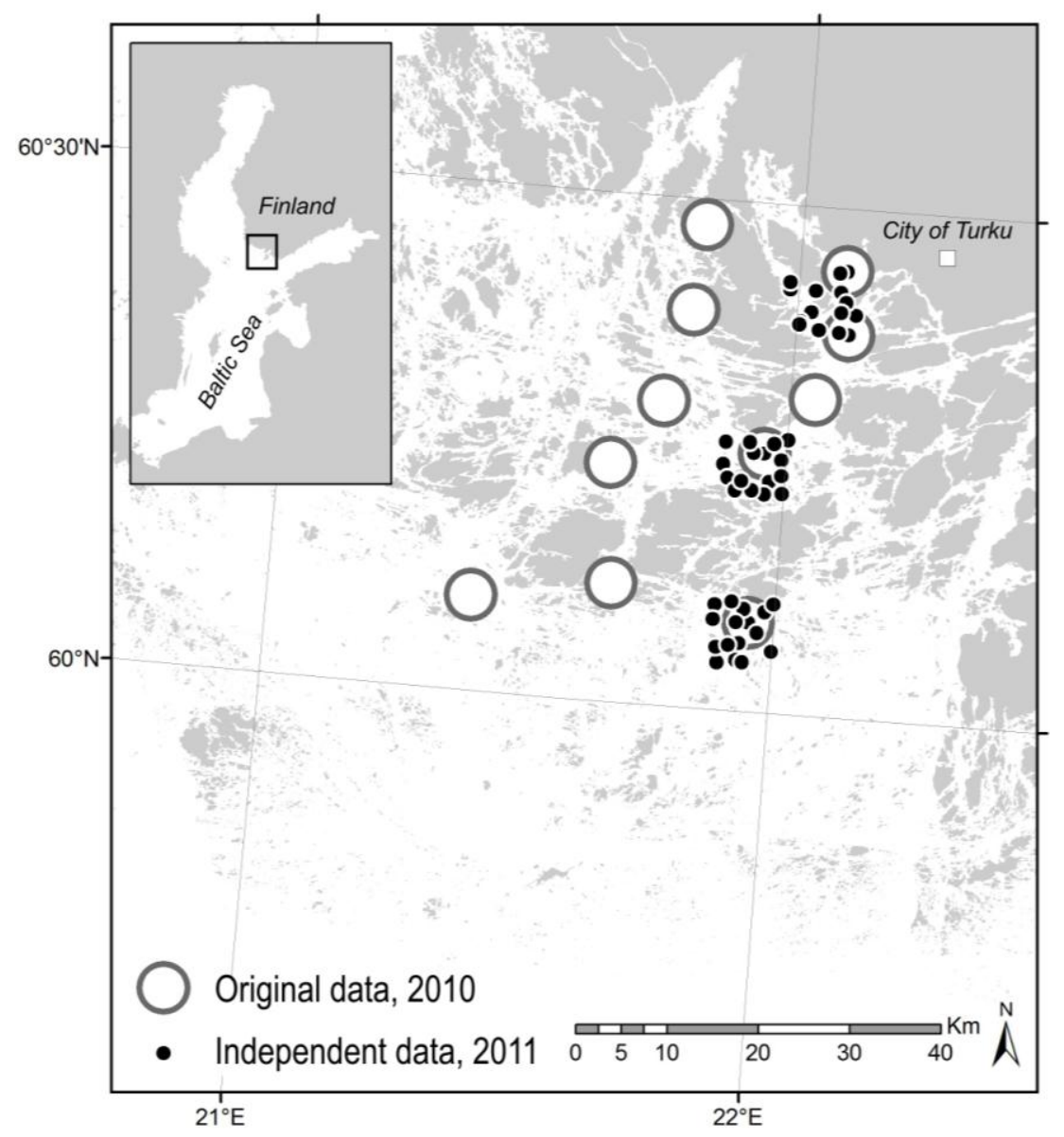

\subsection{Data Processing}

An empirical $m$ coefficient was computed for the $88 Z_{S D}-Z_{\text {eu }}$ pairs in the original dataset. Different categorization methods were applied, and a coefficient for each created category was computed as an average of the $m$ values within the respective group. In addition, a linear function and a power function were fitted. We ended up with 7 locally calibrated conversion methods to be tested: (1) a constant based on average of the whole dataset; (2) 8 coefficients based on measurement weeks (hereafter week method); (3) 11 coefficients based on measurement stations (station method); (4) 3 coefficients based on the archipelago zones (zone method); (5) 4 coefficients based on the water transparency values (quartile method); (6) a linear function; and (7) a power function. The three archipelago zones had been pre-defined by a hierarchical cluster analysis, according to the optical properties of the same original dataset (for the analysis, see [12]). The water transparency classification was created by dividing the $Z_{S D}$ values into quartiles. 
These seven locally calibrated methods were compared with 2 methods derived from literature. The first one is a general coefficient 3, which, as an integer, is convenient to use, and therefore often suggested in literature and used in practice. Secondly, we tested a 2-level water transparency classification introduced by Holmes [7]; a factor of 3.5 for waters with $Z_{S D}$ less than $5 \mathrm{~m}$, and a factor 2 for higher transparencies (hereafter 2-level method). A general baseline of the study was to assess the performance of the eight other methods compared to the performance of the constant 3.

First, the performances of the conversion types were assessed for the original data. The modeled $Z_{e u}$ values were compared with the observed ones, and three error indicator values were derived for each conversion method: mean absolute error (MAE), mean relative error (MRE), and relative root-mean-squared error (RRMSE). Scatter plots representing modeled and observed values were drawn to further illustrate the accuracy of the conversion procedures. The fitting determination coefficients $\left(\mathrm{R}^{2}\right)$ were added to the plots.

The second step was to assess the modeling accuracy of the same methods, except the one based on stations, with an independent dataset. Instead of calibrating the methods again, $Z_{e u}$ values were modeled by using the coefficients and functions defined with the training data in the first phase. The modeling result was assessed as successful if the modeled value deviated one meter or less from the observed value. The relative share of the successful cases then indicates the overall success rate of the method. The same was repeated with the accuracy limit of $2 \mathrm{~m}$. The results for models based on the constant $3, Z_{S D}$ quartiles, and the power function were mapped for illustrating the spatio-temporal dynamisms of the conversion performance.

\section{Results}

\subsection{Model Building and Testing with the Original Training Data}

The original training data included a total of 88 Secchi depths and light profiles. Within this dataset, $Z_{S D}$ values varied from 0.8 to $7.5 \mathrm{~m}$ (average $3.6 \mathrm{~m}$ ), and $Z_{e u}$ values varied from 2.8 to $18.0 \mathrm{~m}$ (average $9.6 \mathrm{~m}$ ). The $Z_{S D}$ data approached a normal distribution with some uncertainty, as at $5 \%$ significance level, the K-S test implied normality ( $p$-value 0.200 ), and the $S-\mathrm{W}$ test $\operatorname{did}$ not ( $p$-value 0.012 ). The $Z_{S D}$ distribution was somewhat positively skewed (skewness value 0.375 ), indicating that low values were somewhat more abundant than high values, but the sample size was considered to be large enough to sufficiently cover the entire $Z_{S D}$ range. The $Z_{e u}$ values were normally distributed according to both statistical tests.

In general, the $Z_{e u}$ values increased in the transition from the inner archipelago towards the open sea areas of the Baltic Proper. However, there were anomalies in this pattern, especially some notable temporal differences. The $Z_{e u}$ values followed largely a seasonal fluctuation pattern but the exact timing of the phases in the seasonal development varied considerably among archipelago areas. That is, the peaks and troughs of the sampling stations did not coincide (see also [12]).

The linear correlation between $Z_{S D}$ and $Z_{e u}$ was very strong and positive (Pearson 0.941), and the coefficient of determination was high $\left(\mathrm{R}^{2}=0.8864\right)$. The correlation between the measured $Z_{S D}$ and the ratio of $Z_{e u}$ to $Z_{S D}$-i.e., the empirical coefficient $m$-was a bit weaker but negative (Figure 2). All three results were statistically significant $(p$-value $<0.001)$. The empirical coefficient, $m$, changes 
according to the overall clarity of water: the greater the water transparency, the lower the factor between $Z_{S D}$ and $Z_{e u}$.

Figure 2. Correlation (Pearson) between (A) Secchi depth $\left(Z_{S D}\right)$ and euphotic depth $\left(Z_{e u}\right)$; and (B) Secchi depth and the ratio of these two. The depths are measured values of the original dataset.

A

$$
Z_{\text {eu }}(m)
$$

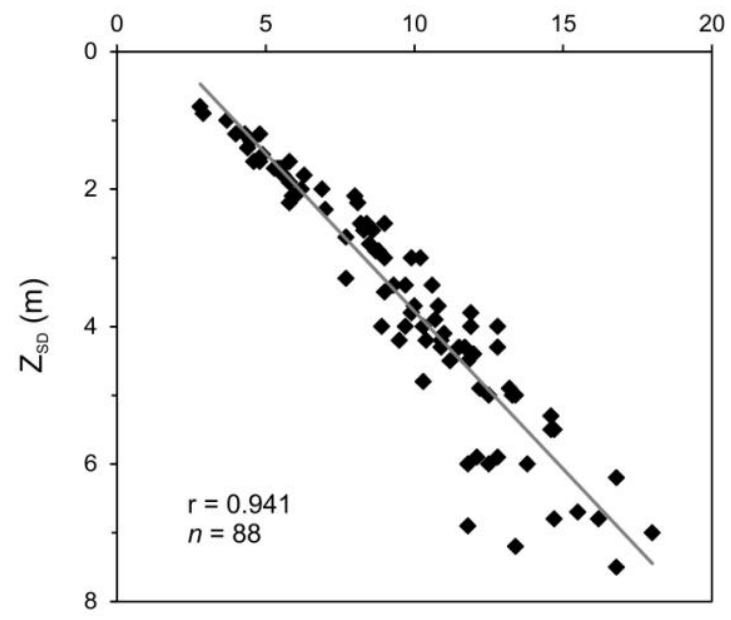

B $Z_{\text {eu }}: Z_{\mathrm{sD}}$ ratio

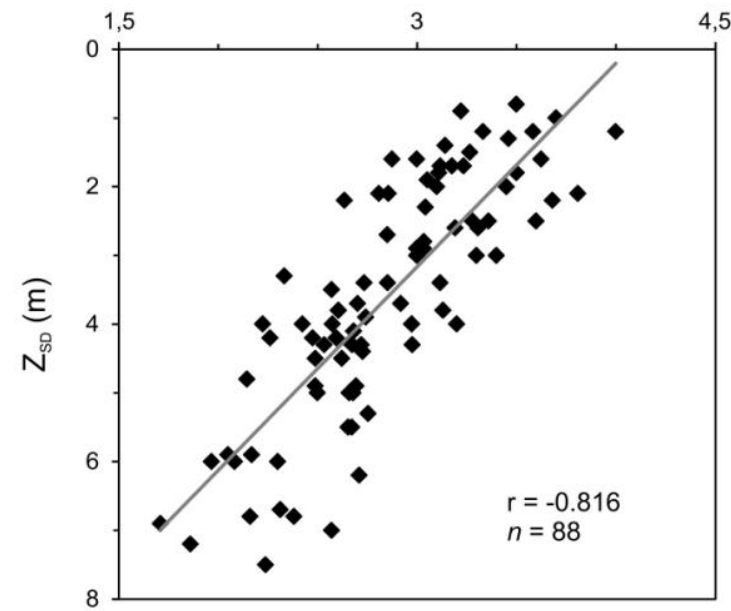

First we modeled $Z_{e u}$ values with a fixed coefficient 3, for which MAE was $1.7 \mathrm{~m}$ and MRE 15.5\%. High $Z_{e u}$ values were exaggerated, and low $Z_{\text {eu }}$ values were underestimated (Figure 3A). The range of error varied from $8.9 \mathrm{~m}$ exaggeration to $1.7 \mathrm{~m}$ underestimation. Secondly, we tested a fixed coefficient optimized for this training set. The average of 88 coefficients in the set was 2.85 (median 2.83), and by using the average instead of the integer, the MAE was decreased from $1.7 \mathrm{~m}$ to $1.4 \mathrm{~m}$, and the RRMSE from $20.9 \%$ to $18.9 \%$ (Table 1 ).

Table 1. The error indicators for the tested models.

\begin{tabular}{cccc}
\hline \multirow{2}{*}{ Method } & $\begin{array}{c}\text { MAE } \\
\text { m }\end{array}$ & $\begin{array}{c}\text { MRE } \\
\text { \% }\end{array}$ & $\begin{array}{c}\text { RRMSE } \\
\text { \% }\end{array}$ \\
\hline Constant 3 & 1.7 & 15.5 & 20.9 \\
Constant 2.85 & 1.4 & 14.2 & 18.4 \\
Week & 1.3 & 13.3 & 17.0 \\
Station & 1.0 & 10.3 & 12.9 \\
Zone & 1.1 & 11.2 & 14.1 \\
2-Level & 2.0 & 19.5 & 24.0 \\
Quartile & 0.9 & 8.7 & 11.1 \\
Linear function & 0.9 & 10.8 & 14.3 \\
Power function & 0.8 & 8.5 & 10.7 \\
\hline
\end{tabular}


Figure 3. The observed $Z_{e u}$ values plotted against the predicted values that are modeled by the nine methods tested in this study: (A) by constant 3; (B) average constant 2.85; (C) week method; (D) station method; (E) zone method; (F) 2-level method; (G) quartile method; (H) linear function; and (I) power function. The black 1:1 lines indicate perfect correlations, and the red dashed lines are trendlines related to the coefficients of determination shown on the corners. For each case, $n=88$.

A

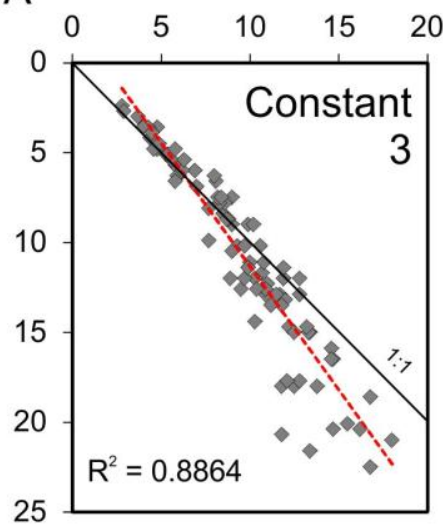

$\mathrm{D}$

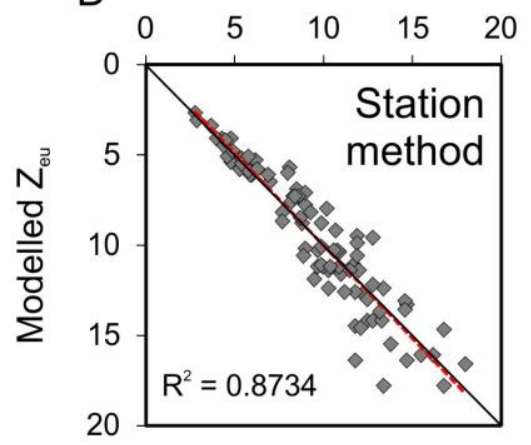

G

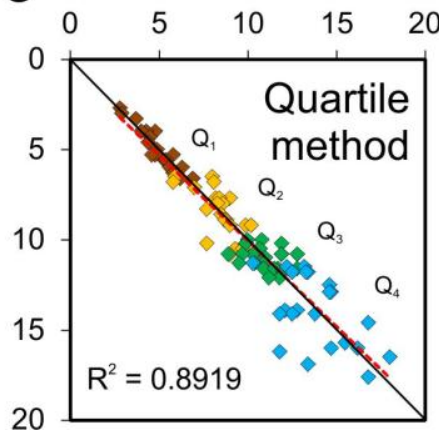

B

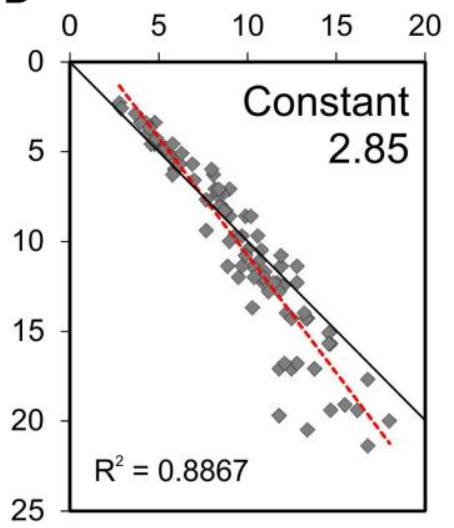

E

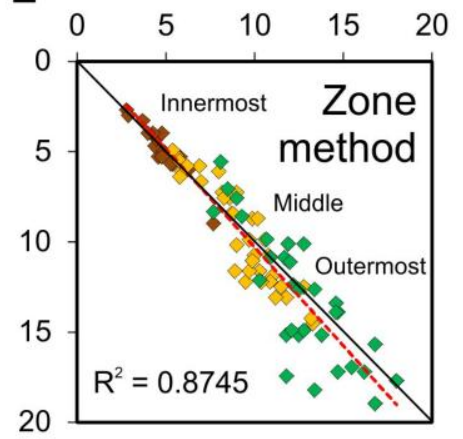

$\mathrm{H}$

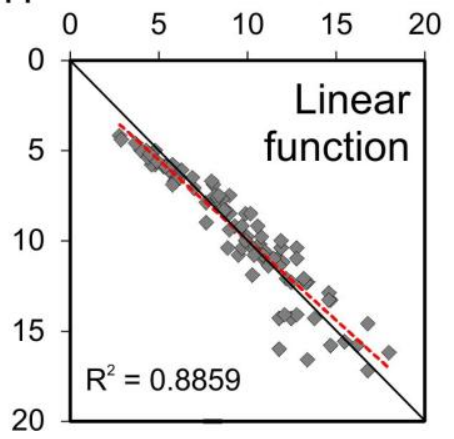

C

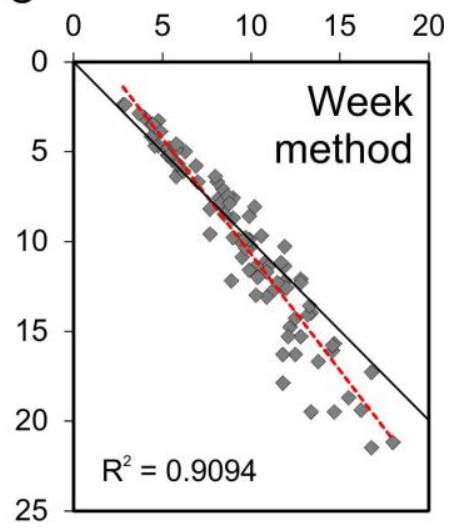

$\mathrm{F}$

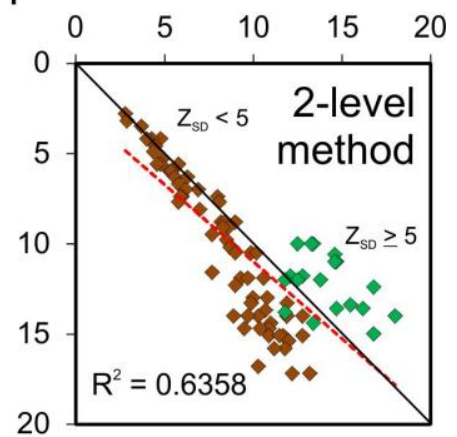

I

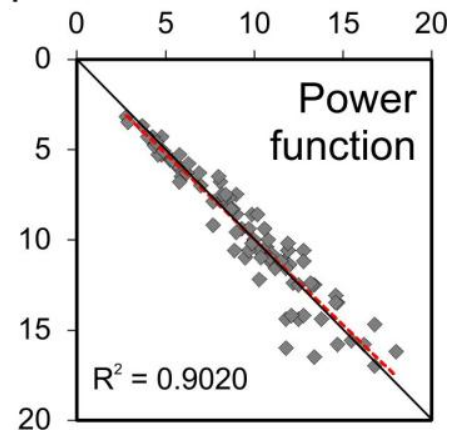

We divided the $Z_{S D}-Z_{e u}$ observation pairs to smaller groups in order to study the coefficients in more detail. The $m$ coefficients calculated for the station method varied from 2.43 to 3.43 , whereas, in the inner, middle, and outer archipelago of the zone method they averaged 3.33, 2.87, and 2.48, respectively. The coefficients defined by the measurement weeks ranged from 2.60 (week 34) to 3.05 (week 31). In all three cases, the modeled $Z_{e u}$ values were a bit more accurate than when using a single, fixed coefficient, but the mean errors (MAE and MRE) still remained greater than $1 \mathrm{~m}$ or $10 \%$. The week method performed the poorest, and the station method only somewhat better than the zonal one (Table 1). 
Further comparisons can be made with scatter plots, which reveal rather similar problems for $Z_{e u}$ values modeled with week method than for those modeled with one constant; high $Z_{e u}$ values are greatly exaggerated (Figure $3 \mathrm{C}$ ). Even though the $\mathrm{R}^{2}$ value is the highest of all of them, the error values also remain high as the trend is clearly biased. Some improvements in the prediction accuracy in clear waters were gained with the zone method (Figure 3E), and further improvements with the station method (Figure 3D). Nevertheless, most of the high values still remain below the 1:1 line.

Next, we tested the 2-level method suggested by Holmes [7]. The accuracy of conversion actually decreased when using two coefficients ( 3.5 for $Z_{S D}<5 \mathrm{~m}$, and 2 for $Z_{S D} \geq 5 \mathrm{~m}$ ) instead of one (Table 1). The scatter plot shows a clear cut between the water transparency classes, and consequently, the $\mathrm{R}^{2}$ remains much lower compared to those of the other methods (Figure 3F).

The water transparency classification based on $Z_{S D}$ quartiles (quartile method) was calibrated with our own data and performed much better. The coefficients calculated for the classes were 3.32, 3.08, 2.69 , and 2.35 starting from the quartile of the lowest water transparency values towards clearer waters (Table 2). The scatter plot was more evenly distributed around the 1:1 line than in most of the cases, which links the observed and modeled values more closely together (Figure 3G). The improved accuracy is also apparent in the error indicators (Table 1). For example, the derived $Z_{e u}$ values deviated on average $0.9 \mathrm{~m}$ from the observed ones (MAE), which corresponds to MRE less than $10 \%$.

Table 2. The 88 Secchi depths were divided into quartiles and an empirical coefficient $m$ was derived for each of the quartile by calculating an average of the $Z_{e u}: Z_{S D}$ ratios within the respective group.

\begin{tabular}{ccc}
\hline Category & Secchi Depth & $\boldsymbol{m}$ \\
\hline $\mathrm{Q}_{1}$ & $<2.1 \mathrm{~m}$ & 3.32 \\
$\mathrm{Q}_{2}$ & $2.1-3.6 \mathrm{~m}$ & 3.08 \\
$\mathrm{Q}_{3}$ & $3.7-4.5 \mathrm{~m}$ & 2.69 \\
$\mathrm{Q}_{4}$ & $>4.5 \mathrm{~m}$ & 2.35 \\
\hline
\end{tabular}

The $Z_{S D}$ values were treated as individual values, independent on their measurement location and timing. This means that the readings from one particular station were not always categorized into the same water transparency class, but the optimal conversion coefficient often changed among the measurement weeks during the growing season. Only one out of 11 stations remained in the same water transparency category, and as many as eight fluctuated among three out of four categories. Moreover, during six out of eight measurement weeks, there was at least one $Z_{S D}$ reading within all the water transparency classes. Only during the periods of low light availability (weeks 17 and 31), the $Z_{S D}$ conversion coefficient for the clearest waters was left unused.

Finally, a linear and a power function were derived to connect the two parameters. The previous resulted as

$$
Z_{e u}=1.9322 \times Z_{S D}+2.6629
$$

and the latter as

$$
Z_{\text {eu }}=3.7489 \times Z_{S D}^{0.7506}
$$


In general, the functions performed better than the fixed or scalable coefficients used in the conversion. The MAE remained under $1 \mathrm{~m}$, as it did with the quartile method. The power function performed slightly better than the linear one, and actually resulted as the best option compared to all the tested methods (Table 1). However, the differences between the results of the functions and the quartile method are relatively small. The scatter plots of the power function and the quartile based conversions were rather similar, but the linear function seemed to exaggerate $Z_{e u}$ values in turbid waters (Figure 3). Statistically, the averages of the modeled $Z_{e u}$ values, regardless of the conversion type, did not differ from the average of the observed values at $5 \%$ significance level.

\subsection{Applicability Testing with the Independent Testing Data}

Besides assessing the performance of the conversion methods with the training data, we wanted to test their modeling accuracy with an independent dataset. The results were varying. Within our testing data, the functions performed the best as they predicted more than $70 \%$ of the euphotic depths within the set limit of $1 \mathrm{~m}$. As both the constants, also the zone, week, and 2-level method reached a success rate less than $40 \%$, whereas the quartile method succeeded in more than half of the cases. By increasing the confidence limit to $2 \mathrm{~m}$, all the success rates grew to be greater than $50 \%$, except for the 2-level method, which performed the poorest. For the quartile method and the functions, almost all the modeled $Z_{e u}$ values fitted within the $2 \mathrm{~m}$ limit as the success rates rose above $90 \%$ (Table 3).

Table 3. The results of the applicability testing with the independent dataset of 2011. The relative shares $(\%)$ of the modeled $Z_{e u}$ values deviating maximum of $1 \mathrm{~m}$ or $2 \mathrm{~m}$ from the observed $Z_{e u}$ values, $n=94$.

\begin{tabular}{ccc}
\hline & $\mathbf{1 ~ m}$ & $\mathbf{2 ~} \mathbf{~ m}$ \\
\hline Constant 3 & 27.7 & 53.2 \\
Constant 2.85 & 39.4 & 62.8 \\
Week & 28.7 & 51.1 \\
Zone & 35.1 & 59.6 \\
2-level & 18.1 & 43.6 \\
Quartile & 57.4 & 91.5 \\
Linear function & 72.3 & 94.7 \\
Power function & 70.2 & 95.7 \\
\hline
\end{tabular}

There was, however, great temporal and spatial variation in the success rates. Some conversion types performed better in early summer (e.g., quartile method), and some in late summer (e.g., constants and functions) (Figure 4). The performance of the constant factors varied considerably between the test areas, whereas the quartile method and the functions achieved more stable results throughout the archipelago zones. However, there was a general trend where success rates of conversion increased towards the mainland in week 23, and towards the outer archipelago in week 31. 
Figure 4. The applicability of two conversion methods locally calibrated in this study were tested with independent data, and the performance was compared with that of a common fixed coefficient 3 . The differences between the modeled and observed $Z_{e u}$ values are plotted on maps. The figure illustrates spatio-temporal variability in the conversion accuracy among three test areas located in different parts of the optically varying coastal archipelago area, and between two timings representing different phases of the seasonal variations in the optical properties.

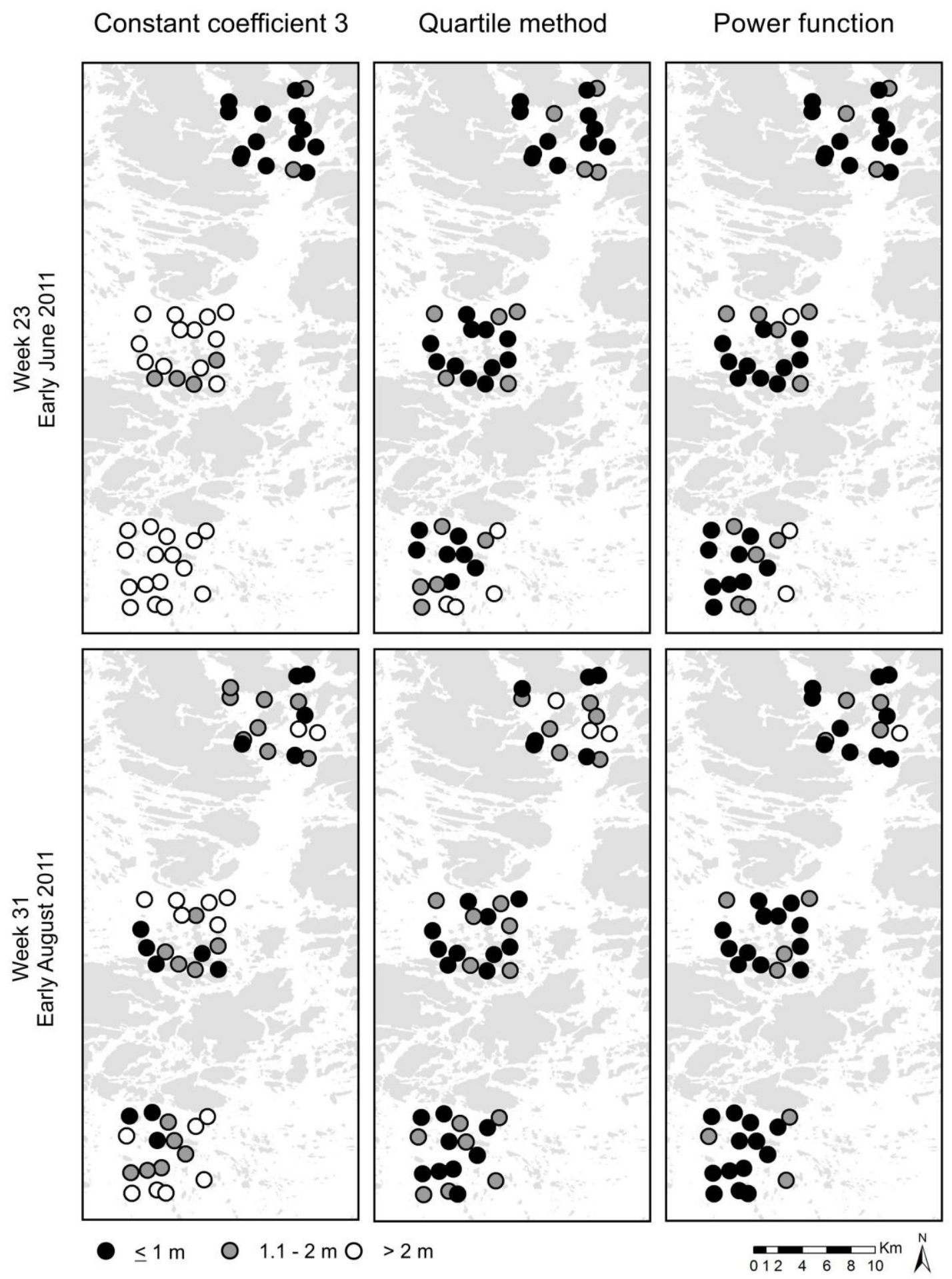




\section{Discussion}

The aim of the study was to compare methods for determining the coefficients between $Z_{S D}$ and $Z_{e u}$. We assessed different criteria to base the coefficient on, and managed to significantly improve the accuracy - in this case to halve the mean absolute error of the original training data, and to double the success rate for the independent testing data - by choosing a more appropriate conversion method than a fixed constant coefficient. The best results were achieved with the function methods that performed well in the accuracy tests of both of the datasets.

As suggested in literature, and supported by our data, the conversion coefficient changes as a function of water transparency change. The conversion functions process this transition as a continuum, and they do not require the sometimes problematic procedure of pre-classifying the data. According to the error indicators, the power function is the best performing conversion method in our study - even though there were only minor differences in the performance among the most accurate methods. Furthermore, the scatter plot illustrating the modeling accuracy of the power function revealed a relatively good fit of model throughout the entire data range. The same applies for the linear function in high $Z_{e u}$ values, but in less transparent water, the values were notably exaggerated.

The other option, besides using functions, is to use fixed or scalable coefficients. They, however, may be problematic, as always when compressing data into averages, some information is lost. Therefore, the values closer to the mean of the respective group are more accurate than the values approaching the limits of the class. Conversions based on averaged coefficients tend to overestimate the modeled $Z_{e u}$ values in clearer waters, and underestimate them in less transparent waters. This is most clearly seen when using only one fixed coefficient, but the phenomenon is also relevant with the scalable coefficients that divide the input data into subgroups - despite the absolute data ranges of the classes. Furthermore, the limiting values are always artificial, whether they are drawn based on pure mathematics, or on the estimates by the researcher - no matter how well established they are.

$\mathrm{Xu}$ et al. [16] stated in their studies - where they tried to estimate the connection between Secchi depth and attenuation coefficient in the Chesapeake Bay - that the bulk relationships fitted over many months and large areas possibly fail because the light controlling dependences change considerably in time and space. Likewise, this applies to our study area, where the optical properties of the less transparent inner archipelago waters and the clearer outer archipelago waters differ notably, and the seasonal differences are also distinctive [12,15].

These extremes in water quality can be separated by dividing the area geographically into fixed zones. A more flexible and efficient baseline is to divide the data according to water quality itself. A pre-classification of the original $Z_{S D}$ data before the actual conversion procedure has been suggested before. The 2-level water transparency division suggested by Holmes [7] already in the early 70s was tested in this study, but the results for our dataset were poor. We found the difference between the two coefficient values rather notable, causing a great gap between the modeled values around the border of the two classes. In a recent study by Liu et al. [17] the NTD675 water classification method was used as a baseline for pre-classification to allow more precise assessment of optical properties of water. However, few researchers who convert $Z_{S D}$ to $Z_{e u}$ have access to such data or instruments. This highlights the convenience of the $Z_{S D}$-based pre-classification method, the quartile method, as, after the calibration procedure, no other data or instruments are needed beside the $Z_{S D}$ values themselves. 
The functions do not require any decision-making in the execution phase of the conversion procedure, and thus their usage is rather unambiguous. Likewise, when using the quartile method, it is straightforward to select the appropriate coefficient according to the quartile in which the $Z_{S D}$ reading in question belongs to. These properties are advantageous, for example, when sampling transitional areas between coastal zones. In other words, one avoids the problem of choosing an appropriate zonal coefficient and consequently a formation of imaginary boundaries into sea areas which actually face gradual changes in water quality.

Furthermore, the conversion procedure ought to be flexible when the water quality does not remain stable. High spatio-temporal variability might be left unrecognized by using coefficients that are fixed for timings or locations, such as measurement weeks, stations, or the archipelago zones. They on the other hand require either locally or temporally restricted calibration procedures, but at the same time, they face the bulk problem on the other dimension. In contrast, the quartile method avoids the bulk problem in both of the dimensions. By choosing an appropriate conversion coefficient according to the water transparency, more flexibility is allowed for the procedure as certain areas or timings are not permanently fixed with certain coefficients. Actually, our results show that this flexibility is valuable, as for all the original stations - with only one exception-several of the four coefficients based on $Z_{S D}$ quartiles were used at different occasions during the growing season.

The accuracy of all the models depends on the success of their calibration. The coverage of the calibration data needs to be sufficient and suitable for the purpose. Preisendorfer [3] found the empirically defined conversion coefficients frustrating as the statistical links between the Secchi and euphotic depth may dissolve in time. He rationalized that even though the changes in water quality tend to be periodic, there is always some randomness caused by, for example, climatic or anthropogenic factors, which changes the optical composition of water. Due to the random variability in water quality, the pre-defined conversion coefficients may not be trustworthy after completing the calibration.

Consequently, we urge the use of spatio-temporally comprehensive data when calibrating the coefficients. Including as wide and as variable data as possible in the calibration phase, the coefficients are more likely to perform well also in situations outside the calibration procedure. The link may well be untrustworthy if the coefficients are created based on data collected within a narrow time window, for example during cyanobacteria blooms, and if these coefficients are then used during the water transparency maximum. Instead, our data cover variation in both time and space, and thus the spatio-temporal differences are already included in the coefficients.

Nonetheless, there were spatio-temporal differences in the quality assessment made with the independent testing data (from year 2011). During the first test week, the accuracy improved towards the mainland, while in the second week, it improved towards the open sea. One possible explanation for the poorer performance in the outer archipelago during the first week is the high transparency of waters. Some of the $Z_{S D}$ values in 2011 were notably greater than any $Z_{S D}$ values in 2010, and thus fell outside the original training data range. The quartile method presumably requires a new water transparency class for waters clearer than the ones used in our current classification. The functions, on the other hand, performed better as they rather accurately succeeded in extrapolating the $Z_{S D^{-}} Z_{e u}$ correlation also outside the original data range. 
In our study, the method based on water transparency quartiles modeled the $Z_{e u}$ values in the original training data very well, and in the independent testing data with moderate accuracy. The method is assumed to work with sufficient accuracy whenever the calibration data, i.e., the computed coefficients, are derived at the same sea area, or at least from optically similar waters - it is not necessary to use exactly the same locations. Nevertheless, it is important to consider what kind of waters are in question. Coefficients defined in very turbid waters will presumably not work well in optically very different waters, such as very clear waters, or waters in which the attenuation is strongly dominated by organic material.

As geographical information is increasingly demanded in coastal research and administration, also euphotic depth data are needed. The applications for spatial $Z_{\text {eu }}$ data range from studies of phytoplankton and macrophyte ecology to the planning work related to integrated coastal zone management (ICZM) and marine spatial planning (MSP). In each case, it is crucial to operate with data which reflect the true euphotic depth as well as possible. Instead of using one or few measured $Z_{S D}$ values to represent a certain coastal area, we urge to study the area comprehensively. As a part of this, an optimized $Z_{S D}$ to $Z_{e u}$ conversion will often notably improve the results of the coastal GIS work.

\section{Conclusions}

We suggest sufficient attention to be paid towards the methodology when converting Secchi observations to estimates of the euphotic depth - especially in waters, where the optical properties are poorly known. When $Z_{S D^{-}}$derived euphotic depths are used in, for instance, spatial models of marine ecosystems, deviations in the conversion may propagate significant modeling errors. The highest accuracy can be achieved only by using locally calibrated conversion methods. In this study, we managed to halve the mean errors of modeling accuracy by using locally calibrated conversion functions or scalable $Z_{S D}$-quartile based conversion coefficients instead of a fixed coefficient. However, there are situations where no calibration data are available. If a conversion method optimized elsewhere needs to be applied, coefficients calibrated in optically as similar waters as possible should be selected. Furthermore, we consider methods that are based on water transparency $\left(Z_{S D}\right)$ values - either continuous functions or series of constants - a more suitable approach to the conversion procedure than using coefficients fixed with certain locations or time periods as optical properties may vary considerably in space and time. Regardless of the method selection, the euphotic depth remains a crucial and spatio-temporally fluctuating variable of the coastal environment. Thus, its inclusion in models and decision support systems is both a challenge and a manageable task for spatial data management in coastal GIS.

\section{Acknowledgments}

The study was financially supported by Kone Foundation, EU Life+ (FINMARINET project), and the Academy of Finland (project 251806). We are grateful to Risto Kalliola for fruitful discussions and valuable comments on the manuscript, and for assistance with the in situ sampling. We would also like to thank Timo Rantanen for his help in the data collection. 


\section{Conflicts of Interest}

The authors declare no conflict of interest.

\section{References}

1. Kirk, J.T.O. Light and Photosynthesis in Aquatic Ecosystems, 3rd ed.; Cambridge University Press: Cambridge, UK, 2011.

2. Lee, Z.; Weidemann, A.; Kindle, J.; Arnone, R.; Carder, K.L.; Davis, C. Euphotic zone depth: Its derivation and implication to ocean-color remote sensing. J. Geophys. Res. 2007, 112, C03009:1-C03009:11.

3. Preisendorfer, R.W. Secchi disk science: Visual optics of natural waters. Limnol. Oceanogr. 1986, 31, 909-926.

4. Koenings, J.P.; Edmundson, J.A. Secchi disk and photometer estimates of light regimes in Alaskan lakes: Effects of yellow color and turbidity. Limnol. Oceanogr. 1991, 36, 91-105.

5. Swan, B.K.; Reifel, K.M.; Tiffany, M.A.; Watts, J.M.; Hurlbert, S.H. Spatial and temporal patterns of transparency and light attenuation in the Salton Sea, California, 1997-1999. Lake Reserv. Manage. 2007, 23, 653-662.

6. Padial, A.A.; Thomaz, S.M. Prediction of the light attenuation coefficient through the Secchi disk depth: Empirical modeling in two large Neotropical ecosystems. Limnology 2008, 9, 143-151.

7. Holmes, R.W. The Secchi disk in turbid coastal waters. Limnol. Oceanogr. 1970, 15, 688-694.

8. Aarup, T. Transparency of the North Sea and Baltic Sea-A Secchi depth data mining study. Oceanologia 2002, 44, 323-337.

9. Montes-Hugo, M.A.; Alvarez-Borrego, S.; Giles-Guzmán, A.D. Horizontal sighting range and Secchi depth as estimators of underwater PAR attenuation in a coastal lagoon. Estuaries 2003, 26, 1302-1309.

10. Tolvanen, H.; Suominen, T.; Kalliola, R. Annual and long-term water transparency variations and the consequent seafloor illumination dynamics in the Baltic Sea archipelago coast of SW Finland. Boreal Environ. Res. 2013, 18, 446-458.

11. Stramska, M.; Frye, D. Dependence of apparent optical properties on solar altitude: Experimental results based on mooring data collected in the Sargasso Sea. J. Geophys. Res. 1997, 102, $15679-15691$.

12. Luhtala, H.; Tolvanen, H.; Kalliola, R. Annual spatio-temporal variation of the euphotic depth in the SW-Finnish archipelago, Baltic Sea. Oceanologia 2013, 55, 359-373.

13. Kratzer, S.; Håkansson, B.; Sahlin, C. Assessing Secchi and photic zone depth in the Baltic Sea from satellite data. Ambio 2003, 32, 577-585.

14. Darecki, M.; Stramski, D. An evaluation of MODIS and SeaWiFS bio-optical algorithms in the Baltic Sea. Remote Sens. Environ. 2004, 89, 326-350.

15. Suominen, T.; Tolvanen, H.; Kalliola, R. Geographical persistence of surface-layer water properties in the Archipelago Sea, SW Finland. Fennia 2010, 188, 179-196.

16. Xu, J.; Hood, R.R.; Chao, S.-Y. A simple empirical optical model for simulating light attenuation variability in a partially mixed estuary. Estuaries 2005, 28, 572-580. 
17. Liu, J.; Sun, D.; Zhang, Y.; Li, Y. Pre-classification improves relationships between water clarity, light attenuation, and suspended particulates in turbid inland waters. Hydrobiologia 2013, 711, 71-86.

(C) 2013 by the authors; licensee MDPI, Basel, Switzerland. This article is an open access article distributed under the terms and conditions of the Creative Commons Attribution license (http://creativecommons.org/licenses/by/3.0/). 